\title{
Filigrane
}

Écoutes psychanalytiques

\section{Les dispositifs d'intervention clinique en institution}

\section{Jean-Pierre Pinel}

Volume 24, numéro 2, automne 2015

Le devenir de la psychanalyse. Que nous disent les pratiques institutionnelles?

URI : https://id.erudit.org/iderudit/1036534ar

DOI : https://doi.org/10.7202/1036534ar

Aller au sommaire du numéro

Éditeur(s)

Revue Santé mentale au Québec

ISSN

1192-1412 (imprimé)

1911-4656 (numérique)

Découvrir la revue

Citer cet article

Pinel, J.-P. (2015). Les dispositifs d'intervention clinique en institution.

Filigrane, 24(2), 109-116. https://doi.org/10.7202/1036534ar

\section{Résumé de l'article}

Cet article présente de manière synthétique la théorisation et la découpe originales de l'intervention clinique en institution en référence au modèle que Jean-Claude Rouchy nous a proposé. Il présente et discute trois repères essentiels à la compréhension de sa démarche méthodologique : le concept de dispositif ; la place de l'analyste ; les différents dispositifs d'analyse en institution. Ce modèle offre un repérage particulièrement précieux pour le clinicien, trouvant ainsi un adossement fiable et consistant lui permettant de résister créativement à la déliaison, voire au chaos, qu'il rencontre de plus en plus massivement dans sa clinique d'intervention, soumise aux bouleversements du méta-cadre contemporain. 


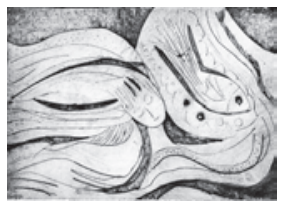

\title{
Les dispositifs d'intervention clinique en institution ${ }^{1}$
}

\author{
Jean-Pierre Pinel
}

Cet article présente de manière synthétique la théorisation et la découpe originales de l'intervention clinique en institution en référence au modèle que JeanClaude Rouchy nous a proposé. Il présente et discute trois repères essentiels à la compréhension de sa démarche méthodologique: le concept de dispositif; la place de l'analyste; les différents dispositifs d'analyse en institution. Ce modèle offre un repérage particulièrement précieux pour le clinicien, trouvant ainsi un adossement fiable et consistant lui permettant de résister créativement à la déliaison, voire au chaos, qu'il rencontre de plus en plus massivement dans sa clinique d'intervention, soumise aux bouleversements du méta-cadre contemporain.

et article se donne pour finalité de présenter de manière synthétique la théorisation et la découpe originales de l'intervention clinique en institution en référence au modèle que Jean-Claude Rouchy nous a proposé. Le propos s'appuie essentiellement sur l'ouvrage intitulé «Institution et changement» que Jean-Claude Rouchy a publié en co-écriture avec Monique Soula Desroche (2004). Il présentera et discutera trois repères essentiels à la compréhension de sa démarche méthodologique: le concept de dispositif, la place de l'analyste, les différents dispositifs d'analyse en institution.

\section{Le concept de dispositif}

C'est un concept essentiel dans la théorisation proposée par Jean-Claude Rouchy, fermement distingué de celui de cadre institutionnel. Le dispositif est caractérisé par deux éléments princeps: d'une part, il doit être différent de celui du cadre de travail habituel mis en place par la structure, et d'autre part, il ne doit pas faire écran aux événements.

C'est en effet dans cet écart que pourra se créer potentiellement un site pour l'analyse. Le décalage introduit par le dispositif autorise une différenciation et partant, favorise un travail de symbolisation des pratiques quotidiennes, du faire habituel, souvent automatisé, c'est-à-dire intériorisé sur le mode de l'incorporat (Rouchy, 1998). L'écart permet que le dispositif 
advienne comme contenant temporaire du cadre institutionnel et de celui de l'équipe instituée. L'instauration de cet écart va d'ailleurs fréquemment mobiliser d'emblée différentes formes de résistance, tendant à assimiler le dispositif au cadre de travail, à réduire la distance au profit du retour à l'identique, ce qui constitue une des formes princeps de Thanatos en institution.

D'autre part, le dispositif ne doit pas être trop distant de la réalité quotidienne, c'est-à-dire qu'il doit être relié à la vie institutionnelle, aux pratiques, aux événements et aux situations rencontrées par les praticiens. Aucun dispositif ne peut être préfabriqué, il est toujours construit en rapport avec un établissement ou un service concret articulé aux singularités des demandes adressées à l'intervenant. Le dispositif est à la fois le support du lien contractuel dans la réalité de l'espace d'analyse, et le lieu du transfert, où ce qui se dit et se produit peut être interprété. Le dispositif est déterminé par l'analyste comme étant la structure dans laquelle les relations entre les personnes et les groupes vont prendre place, en rapport avec leurs groupes d'appartenance et leurs réseaux d'interactions intériorisés. Le dispositif est aussi une modalité de contre-transfert anticipé (Rouchy, 1998) dans lequel l'analyste loge des éléments narcissiques, des éléments de ses groupes internes, de ses idéaux et sans doute les avatars et les zones d'ombre de son parcours de formation (Pinel, 1997).

De façon opératoire, un dispositif est constitué d'éléments qui délimitent le rapport au temps, à l'espace et à l'objet du travail. Il y a une dialectique entre cadre institutionnel et dispositif d'analyse: le cadre institutionnel traverse le dispositif et réciproquement, mobilisant ainsi la dynamique interne des groupes de travail. C'est par cette voie que s'opèrent les remaniements du cadre institutionnel au décours de l'analyse. Espace intermédiaire, le dispositif est le lieu où s'effectue la métabolisation de la réalité psychique de chacun, celle des équipes de travail et celle de la réalité instituée.

\section{La place de l'analyste}

J.C. Rouchy souligne que, avant même que l'analyste intervienne, avant même le premier contact, un processus a déjà été initié, qui devra être pensé et exploré. Cet originaire comprend à tout le moins l'ensemble des mouvements psychiques et des procédures ayant précédé l'énoncé de la demande, toujours pris dans des mouvements transférentiels puissants qui auront à être repris durant certains moments clefs de l'intervention. Ainsi, à titre d'exemple, le choix de l'analyste, de ses appartenances et de ses positions 
théoriques supposées peut venir à la fois répéter et contre-investir les conflits traversant l'équipe.

J.C. Rouchy met aussi l'accent sur une deuxième caractéristique appendue au transfert en situation d'intervention en institution: tout ce qui se passe ou se dit dans le travail d'analyse prend une signification en rapport avec la place réelle ou imaginaire attribuée à l'analyste. L'extériorité n'est qu'apparente. L'analyste est toujours saisi dans les processus qui se développent dans le groupe et il est notamment assigné à différentes places au sein des liens intersubjectifs et de la vie fantasmatique du groupe. Il peut être ainsi appelé à la place d'un personnage central tel qu'un ancien directeur ou, et cela est de plus en plus fréquent, en place de répondant institutionnel, ou encore de refondateur qui va recréer des valeurs instituantes mises à l'épreuve par les transformations actuelles de l'arrière-plan socioculturel, économique et juridique (Pinel, 2008). L'analyse de ces mouvements psychiques vient toujours solliciter puissamment le narcissisme et le contretransfert de l'intervenant, de telle sorte que la conduite de l'analyse en institution suppose l'appui sur un groupe institué d'arrière-plan qui est aussi le lieu d'une intervision ou d'une supervision toujours nécessaire dans ces configurations complexes.

\section{Les différents dispositifs de l'analyse en institution}

Les institutions spécialisées recèlent une fragilité de fond corrélative à une suffisante sensibilité à la vie psychique de l'autre et des autres (Gaillard et Pinel, 2011). Cette fragilité est inhérente à l'exercice de la tâche primaire: elle constitue une condition nécessaire à l'engagement d'un travail psychique et au déploiement d'un processus institutionnel.

Simultanément, cette vulnérabilité est liée à la place occupée par ces établissements qui se sont situés, dès leur fondation, dans une position intermédiaire afin d'exercer une fonction d'articulation entre les institutions ordinaires et les sujets ou les groupes présentant une pathologie ou problématique sociale qui les exclut — temporairement ou durablement — de la trame symbolique partagée. Les injonctions actuelles, liées aux transformations du méta-cadre social et culturel, exigeant qu'elles se conforment au mode de fonctionnement des institutions ordinaires, et plus particulièrement à celui des entreprises, risquent de les déloger de cette position intermédiaire et dès lors, d'altérer leurs capacités articulaires.

Les équipes institutionnelles ont à se confronter sans cesse, du dedans, aux diverses expressions de la déliaison, de la destructivité, de la haine et de la 
désespérance. Elles ont ainsi à se déprendre de la fascination pour l'horreur et de l'aspiration à répéter à l'identique (Michel de M'Uzan, 1977) la problématique des sujets accueillis. Pour accueillir ces mouvements psychiques archaïques et violents, elles ont à se constituer dans une forme de paradoxe de fond qui conjoint une suffisante ouverture aux déformations engendrées par le contact avec les patients et une certaine fermeté positionnelle permettant de rétablir un écart, toujours mis à l'épreuve. La contenance de ces vulnérabilités de fond et le maintien d'une position articulaire dépendent de l'instauration de dispositifs cliniques groupaux de métabolisation des effets dissociatifs ou confusionnants traversant les différents espaces et instances institutionnels. Les effets de la pathologie se conjuguent d'une part aux mouvements de dissociation qui traversent régulièrement les équipes instituées et d'autre part, aux bouleversements qui affectent le cadre institutionnel. La négativité peut se loger en des sites différents et prendre des formes variées qui vont nécessiter de construire un dispositif d'intervention appropriable par les praticiens et l'ensemble qu'ils forment. Jean-Claude Rouchy a donné une intelligibilité à ces différentes configurations et contribué à proposer un modèle d'ensemble rigoureux de l'intervention institutionnelle. Ce modèle offre une méthodologie consistante accordée aux diverses demandes adressées aux intervenants cliniciens, différenciant: l'analyse des pratiques, les régulations d'équipe, l'analyse du cadre institutionnel et l'action-recherche.

\section{L'analyse de la pratique et la supervision}

Très régulièrement, les équipes ne peuvent traiter l'ensemble des matériaux psychiques projetés, déposés ou injectés dans la psyché des praticiens, dans les systèmes de liens, comme dans le cadre institutionnel. Tel le protiste freudien, les professionnels, l'équipe ou l'ensemble de l'institution vont être périodiquement débordés et «intoxiqués » par les mécanismes pathologiques associés aux différentes formes de psychopathologie qu'ils accueillent.

Les demandes d'intervention clinique énoncées par les équipes oeuvrant dans le champ des institutions spécialisées s'originent donc en premier lieu dans ces débords. Certains sujets, notamment ceux qu'il est convenu de caractériser comme des "cas difficiles», vont plus particulièrement susciter la représentation d'une mise en péril, voire d'un échec des fonctions soignantes ou socio-éducatives de l'équipe instituée (Vidal, 1984, 2007). Il s'agit là d'une source essentielle des demandes d'intervention adressées à un tiers externe. Qu'elles se formulent en termes d'analyse de la pratique ou de supervision clinique, elles résultent d'une reconnaissance par l'équipe de 
la nécessité de recourir à un autre, suffisamment différent et distancié des effets de la problématique des patients ou usagers de l'institution. Dans ces configurations, il s'agit d'instaurer ou de restaurer un site d'accueil et de métabolisation de la négativité mobilisée par les pathologies réunies par la tâche primaire institutionnelle telles que la psychose, l'exclusion, la pathologie gérontologique ou encore la délinquance...

\section{Les régulations d'équipe}

Il est des configurations dans lesquelles les violences, les attaques de la liaison et les différentes modalités de projections dont les projections scissionnelles $^{2}$ se sont profondément inscrites dans les liens d'équipe et ont produit une modalité de résonance pathologique et chronique. Des dissensions profondes quant aux valeurs instituantes à soutenir, des antagonismes pratiques et théoriques se sont fixés et parfois scellés dans l'idéologisation des positions. Les attaques de la professionnalité ont produit des blessures narcissiques profondément inscrites dans les systèmes de liens, de telle sorte que les échanges à propos de la pratique sont radicalement barrés. Des secrets, des effondrements de la confiance ont entravé la possibilité de se parler. Les praticiens ne parviennent plus à se constituer en un groupe de travail au sens de Bion (1961). Ils ne peuvent, du même coup, construire et mettre en oeuvre des projets et des dispositifs institutionnels suffisamment cohérents, dotés de finalités et de significations partagées. Les instances de réunification du matériel clinique et d'élaboration collective sont immobilisées ou massivement attaquées. L'intervenant est ici requis pour favoriser la réélaboration de liens d'équipe plus vivants et soutenir les capacités de l'équipe à reconstituer un groupe de travail.

En ces configurations, l'offre d'un dispositif d'analyse se formule essentiellement en termes de consultation d'équipe. Jean-Claude Rouchy privilégie ce terme à celui de régulation d'équipe, trop limité à un registre économique, énergétique, délaissant les autres aspects topiques, dynamiques et historiques — participant de la problématique à l'œuvre.

\section{L'analyse du cadre institutionnel}

Dans un troisième type de constellation clinique, l'institution est traversée par une crise qui atteint son cadre, ses fondements matériels, imaginaires et symboliques. Les valeurs, les idéaux, les projets, les modalités de fonctionnement et d'organisation sont attaqués ou vidés de leurs significations. C'est la structure de l'ensemble qui est à mettre collectivement en travail et 
à réélaborer, la pathologie des liens institutionnels s'est étendue à l'ensemble de la structure. Il est cependant à pointer que les institutions les plus profondément prises par la pathologie sont celles qui ont édifié un système de défense si serré qu'elles ne peuvent envisager de recourir à une intervention externe. Méconnaissant ou déniant drastiquement leur fonctionnement pathologique, elles vont se révéler impénétrables. La dureté de ces défenses rend l'institution inutilisable pour les patients. Les résistances collectives se traduisent notamment par un accroissement des exclusions et un recours massif aux prescriptions médicamenteuses afin d'effacer les expressions de la souffrance psychique. Les équipes instituées adressant une demande d'intervention externe témoignent ainsi d'une forme de confiance en l'avenir, d'une espérance suffisamment bonne sur laquelle le dispositif d'intervention prendra appui pour autoriser le réaménagement ou la reconstruction du cadre institutionnel.

Le dispositif, toujours complexe à élaborer, est construit de manière spécifique au regard des singularités des demandes, des problématiques et de la structure concernée. Le dispositif comporte toujours un montage particulier de groupes de travail articulés, dont un groupe de référents, interlocuteur privilégié de l'équipe d'analystes. Vont s'associer des groupes d'analyse des pratiques, des groupes de travail sur le projet, des groupes par fonction, par service dans la confrontation et l'articulation. Un groupe traitant de l'histoire de l'établissement de même que des séances plénières sont instaurés afin de permettre une analyse globale des processus réunissant les expériences vécues par chacun et par les différents groupes de travail, et cela dans une perspective qui allie la synchronie et la diachronie.

\section{Les actions-recherches}

Enfin, il est des demandes adressées à l'intervenant dans lesquelles l'équipe souhaite engager une réflexion approfondie sur le fondement de ses pratiques et ouvrir sur de nouvelles perspectives. Une voie méthodologique féconde est proposée sous la forme d'une action-recherche, terme que Jean-Claude Rouchy privilégie par rapport à celui de recherche-action. Cet ordonnancement indique ici la priorité accordée à la clinique comme source vive de la théorisation. Il est à souligner que ce dispositif est souvent le plus pertinent pour les cliniciens qui sont sollicités pour conduire une recherche ou pour accompagner un changement consistant en interne. La méthode permet de résoudre nombre de paradoxes suscités par l'appartenance partagée entre le clinicien et les équipes. Enfin, l'action-recherche est 
une modalité d'analyse tout à fait appropriée pour développer des pratiques innovantes ou pour étayer la construction de réseaux ou d'appareillage interinstitutionnel. Ce dispositif offre un étayage consistant pour répondre à des situations extrêmement complexes, telles que la mise en ouvre d'un réseau d'ampleur (Rouchy, 1996) ou la construction de dispositifs interinstitutionnels dans l'accompagnement des adolescents en grande difficulté (Soula-Desroche, Rouchy, Pinel, Hemono, 2011).

\section{Conclusion}

Les institutions spécialisées sont actuellement sommées de se transformer sans cesse. Elles sont soumises à de puissantes injonctions économiques, sociales et politiques exigeant qu'elles revisitent de plus en plus fréquemment leur mode de fonctionnement. La logique gestionnaire se traduit par des assauts répétés de refontes des projets de service et d'établissement. Le motif avancé, celui "d'une évaluation garante de qualité», participe fondamentalement à une attaque du travail clinique, à défaire les liens d'équipe et à déstabiliser puissamment les cadres institués. Dès lors, les analystes d'institution sont confrontés à un enchevêtrement de demandes, à une confusion généralisée, produisant un grave trouble dans les équipes instituées. Le modèle que nous propose Jean-Claude Rouchy offre un repérage particulièrement précieux pour le clinicien, trouvant ainsi un adossement fiable et consistant lui permettant de résister créativement à la déliaison, voire au chaos, qu'il rencontre de plus en plus massivement dans sa clinique.

Jean-Pierre Pinel jeanpierre.pinel75@gmail.com

\section{Notes}

1. Cet article reprend l'essentiel d'une communication prononcée lors du colloque international Les fondamentaux de la clinique institutionnelle. D'hier à demain?, Université de Caen, 11-12 Avril 2014.

2. Ce mécanisme repéré et analysé par Paul-Claude Racamier (1980) permet de mieux comprendre les effets dissociatifs ou de scindage mobilisés répétitivement par certains patients.

\section{Références}

Bion, W. R. (1961). Recherches sur les petits groupes. Paris: Presses universitaires des France, 1962.

De M’Uzan, M. (1977). Le même et l'identique. Dans De l'art à la mort (p. 83-97). Paris: Gallimard. 
Gaillard, G. et Pinel, J.-P. (2011). L'analyse de la pratique en institution: un soutien à la professionnalité dans un contexte d'emprise du modèle gestionnaire? Nouvelle Revue de psychosociologie, $\mathrm{n}^{\circ} 11,85-104$.

Pinel, J.-P. (1997). Les phénomènes intercritiques en institutions, impasses et voies potentielles de dépassement. Dans J. Barus-Michel, R. Kaës, et coll., Crises, le sujet à l'épreuve du social. Revigny, France: Hommes et Perspectives.

Pinel, J.-P. (2008). La construction du dispositif d'intervention. Dans O. Nicolle et R. Kaës (dir.), L'institution en héritage (p. 11-24). Paris: Dunod.

Pinel, J.-P. et Gaillard, G. (2013). Les institutions soignantes à l'épreuve de l'hypermodernité. Bulletin de psychologie, 66 (4), 333-340.

Pinel, J.-P. (2014). Le travail de l'intersubjectivité dans les équipes institutionnelles. Dans P. Roman (dir.), L'intersubjectivité en questions. Lausanne: Antipodes.

Racamier, P.-C. (1983). Le psychanalyste sans divan. Paris: Payot.

Rouchy, J.-C. (dir.) (1996). La double rencontre: toxicomanie et sida. Toulouse: Erès.

Rouchy, J.-C. (1998). Le groupe, espace analytique. Toulouse: Érès.

Rouchy, J.-C. (1998). Problématique de l'intervention, Connexions, n 71, 11-27.

Rouchy, J.-C. et Soula Desroche, M. (2004). Institution et changement. Toulouse: Erès.

Soula-Desroche, M., Pinel, J.-P., Rouchy, J.-C. et Hemono, J.-G. (2011). Cadre et dispositif pour la prise en charge des adolescents en grande difficulté. Connexions, n $96,92-105$.

Vidal, J.-P. (1984). De la demande d'intervention analytique dans les institutions. Connexions, 44, Toulouse: Epi.

Vidal, J.-P. (2007). Les « redoublements emboîtés». Le groupe de supervision comme chambre d'échos. Le Divan familial, 19 (2), 141-152. 